\title{
Colorectal neuroendocrine tumors
}

\author{
Beatrice LINTOIU ${ }^{1}$, Irina BALESCU ${ }^{2}$, Nicolae BACALBASA ${ }^{3}$ \\ 1"Profesor Dr. Agripa Ionescu" Clinical Emergency Hospital, Bucharest, Romania \\ 2"Ponderas" Hospital, Bucharest, Romania \\ 3"Carol Davila" University of Medicine and Pharmacy, Bucharest, Romania
}

\begin{abstract}
Gastrointestinal neuroendocrine tumors originate from amine precursor uptake and decarboxylation cells in the digestive tract and have the ability to undergo multiple differentiation and secrete various active hormones, leading to significant differences in biological behaviors and prognosis. Previous studies have indicated a low incidence rate, but recently epidemiological investigations in the USA showed that the incidence rate was significantly higher. Gastrointestinal neuroendocrine tumors have received much attention in recent years with regard to their diagnosis, classification, prognosis and treatment.
\end{abstract}

Keywords: neuroendocrine tumors, gastrointestinal tract, colorectal cancer, carcinoid

\section{INTRODUCTION}

Gastroenteropancreatic neuroendocrine tumors (GEP-NETs), as part of neuroendocrine tumors, are a heterogenous group of tumors derived from neuroendocrine cell compartments in various organs including pancreas, stomach, colorectum and biliary tract. Data from recent population-based studies demonstrate a significant increase in the incidence of NETs over time which ranges from 2.5 to 5 cases per 100000 in Caucasian population. This is attributable to increasing awareness, improved diagnostic strategies for NETs and possibly other undetermined environmental and genetic factors (1). There is a slight overall higher incidence of neuroendocrine tumors for males (5.35) compared with females (4.76). Regarding race, a white predominance $(89 \%)$ has been demonstrated $(2,3)$.

According to the Surveillance, Epidemiology and End results (SEER) database, more than half of all NETs are gastroenteropancreatic NETs (GEP-NETs) (61\%), with the highest frequency being observed in the rectum ( $17.7 \%$ of NETs), small intestine (17.3\% of NETs) and colon (10.1\% of NETs), followed by pancreas (7.0\%), stomach (6.0\%), and appendix (3.1\%) (2).

The new World Health Organization (WHO) Classification of Tumors of the Digestive System presented in 2010 defines the entire group of tumors as neuroendocrine neoplasms and divides them into neuroendocrine tumor (NET), neuroendocrine carcinoma (NEC), mixed adenoneuroendocrine carcinoma (MANEC), hyperplasia and pre-neoplasm. Mixed adenoneuroendocrine carcinoma (MANEC) is morphologically recognizable as both gland-forming epithelial and neuroendocrine phenotype, with each component representing at least $30 \%$ of the lesion. The monomorphous endocrine cells of MANEC have a characteristic of bidirectional differentiation. So, exocrine glandular cells are the key points of MANEC distinguish from other neuroendocrine neoplasms. MANEC are further classified into collision and composite types. Mixed histology tumors are more often found in the appendix and cecum. The poorly differentiated NECs have two subtypes small cell and large cell neoplasms (4). 
The grading system is based on mitotic activity and the percentage of Ki-67 labeled proliferating cells: Grade 1 , grade 2 and grade 3 (= neuroendocrine carcinoma) are defined by mitotic counts of $<2 / 10$ high power fields (HPF), 2-20/10 HPF and > 20/10 HPF, respectively, and/or by Ki- 67 indices of $\leq 2 \%, 3 \%-20 \%$ and $>20 \%$, respectively (4).

The European Neuroendocrine Tumor Society has proposed a tumor-node-metastasis staging and grading system for various types of GEP-NETs (5).

Among neuroendocrine tumors, the ones developing in the colon and the rectum are grouped together in the WHO classification and are distinguished from those of the appendix or the ileum. Colorectal carcinoids are described as "low-grade malignant", even in the presence of metastasis. The WHO classification defines colorectal carcinoids as 'benign' if the tumors measure $20 \mathrm{~mm}$ or less, are localized in the submucosa and have no vascular invasion. Although most colorectal carcinoids are localized at the time of diagnosis and have low malignant potential, rectal carcinoids measuring less than $1 \mathrm{~cm}$ in size still have malignant potential with a recorded incidence of metastasis ranging from 1.7 to $3.4 \%$. Rectal carcinoids are defined as tumors located within $15 \mathrm{~cm}$ of the anal verge, whereas tumors more than $15 \mathrm{~cm}$ above the anal verge are considered colonic carcinoids $(6,7)$.

Extraappendiceal colonic NE tumors, including tumors of the rectosigmoid junction but excluding those of the rectum, are rare malignancies accounting for $1 \%$ of colonic neoplasms, 9.6\% of all NE and $14.1 \%$ of gastrointestinal NE tumors. The predominance of right-sided carcinoid tumors may be due to a greater number of enterochromaffin cells in the right colon, or to the fact that tumors from the appendiceal base extend into the cecum $(3,8)$.

\section{DIAGNOSIS}

Colorectal neuroendocrine tumors grow slowly and are often clinically silent for many years before becoming manifest. They frequently metastasize to the regional lymph nodes, liver, and, less commonly, to the bones. Colonic carcinoid tumors typically present in the seventh decade with nonspecific digestive tract symptoms - abdominal pain, abdominal distention, diarrhea, constipation, anorexia, weight loss or hematochezia $(8,9)$.

Although carcinoids classically are tumors of enterochromaffin and argentaffin cells of the digestive tract, the term carcinoid tumor can be expanded to cover gut tumors of paracrine- and endocrine-like cells of unknown function. It is now established that these tumors have a neuroendocrine origin and derive from a primitive stem cell. They may differentiate into any one of a variety of adult endocrine secreting cells: $\beta$ cell and insulinoma, $\alpha$ cell and glucagono$\mathrm{ma}, \delta$ cell and somatostatinoma, and the PP cell and PPoma, or cells capable of producing ACTH, growth hormone-releasing hormone, VIP, $\mathrm{SP}$, calcitonin, gastrin-releasing factor, Ghrelin, serotonin and the peptide motilin. These cells may secrete one humor at any one point in time whereas at others, the peptide or amine secreted may differ and generate an entirely different clinical syndrome. Metastases are known to secrete hormones that differ from the primary tumor, and different metastases may secrete different hormones $(2,6,10)$.

The carcinoid syndrome, which occurs in less than $10 \%$ of patients with carcinoid tumors, comprises multiple signs and symptoms associated with hypersecretion of vasoactive substances (serotonin, histamine, prostaglandins and tachykinins) by the carcinoid tumor. It includes cutaneous flushing (in $84 \%$ of patients), gastrointestinal hypermotility with diarrhea $(70 \%)$, heart disease (37\%), bronchial constriction (17\%), myopathy (7\%), and an abnormal increase in skin pigmentation (5\%). Urinary 5-HIAA (24-hour collection) is an useful laboratory marker for carcinoid tumors (8-11).

Thus, NETs can be classified into functional or nonfunctional tumors according to the symptoms associated with peptides and hormones production. Carcinoid syndrome, Whipple triad Zollinger-Ellison syndrome, Verner-Morrison syndrome and glucagonoma syndrome are typical symptoms of functional NETs. Most colorectal carcinoids are non-functioning, carcinoid symptomatology being less than $5 \%(12,13)$.

The mean tumor size of colonic carcinoid tumors at presentation has been reported at approximately $5 \mathrm{~cm}$. The cecum is the most frequent location and the tumors are often diagnosed at the late stages of IIIb or IV, with lymph node involvement and distant metastases $(3,8)$. Al Natour and colleagues (14) described a recent trend of increased incidence of smaller and more superficial colonic carcinoid tumors. This is a result of the expanding use of screening colonoscopy, leading to earlier detection of clinically occult colonic lesions. Colonoscopy is the most useful method of diagnosing and treating rectal NETs. Macroscopically, typical NETs ap- 
pear as yellowish, sessile, submucosal tumors, but some have unusual morphology with irregular surfaces or being hyperemic and pedunculated (15).

Definitive diagnosis of GEP-NETs mainly relies on pathological examination. The family of neuroendocrine GEP-NETs, even if it is a heterogenous group, share a common phenotype with immunoreactivity for the pan-neuroendocrine markers including chromogranin A and synaptophysin. Neuroendocrine cells in non-neoplastic and neoplastic tissue of the gastrointestinal tract and nerve elements express a panel of identical antigens, which are used as neuroendocrine markers. The markers chromogranin A, B and C, synaptophysin, HISL-19, proprotein convertases PC2 and PC3, neuron-specific enolase (NSE), lymphoreticular epitope Leu-7 and the neural cell adhesion molecule (CD56) are sufficient to sustain neuroendocrine differentiation independent of hormone production (14-16). The most important of these markers, chromogranin A (CgA), is a 49-kDa acidic polypeptide present in the secretory granules of neuroendocrine cells. Plasma CgA is elevated in $50 \%$ to $100 \%$ of patients with either functioning or nonfunctioning NETs. The sensitivities and specificities of CgA for the detection of NETs range between $70 \%$ and $100 \%$ (17).

A detailed description of the macroscopic, microscopic and immunohistochemical findings is mandatory to support the diagnosis of NETs allowing thus a proper classification, grading and staging. Immunohistochemistry for Ki-67 is mandatory to grade the tumor according to the new WHO classification.

Colonic carcinoids exhibit one of the worst prognoses of all gastrointestinal carcinoid tumors. Prior studies from the 1970s to 1990s reported 5-year survival rates between $23 \%$ and $42 \%$. A more recent analysis of 477 patients from the SEER database reported 5-year survival rates for extraappendiceal colonic carcinoid tumors ranging from $59 \%$ to $87 \%$. The poor prognosis of colonic carcinoid tumors is considered to be a result of the large tumor diameter, higher rates of regional and distant metastatic disease at the time of diagnosis and higher rate of undifferentiated histologic pattern with more aggressive clinical features than carcinoids at other sites $(3,8,13)$.

Patients with a small neoplasm size, shallow invasion, no lymph node metastasis, a low pathological grading and no expression of CgA have a better prognosis. Tumor size was often cited as the most important prognostic indicator for carcinoid tumors, but the best indicators are the evidence of invasive growth and the presence of regional or distant metastasis. Colonic carcinoids exhibit one of the highest rates of nonlocalized disease at presentation of all carcinoid tumors with an overall rate of lymph node metastasis of $48 \%$ to $72 \%$ and distant metastasis present at the time of diagnosis in $25 \%$ to $31 \%$ of the patients. $(9,13,14)$

Given this propensity for metastatic spread, widely accepted consensus guidelines recommend a formal segmental colectomy with oncologic resection of the lymph drainage. Isolated hepatic metastases should be resected if possible $(14,18,19)$. Rectal carcinoids have a risk of lymph node metastasis and distant metastasis varies depending on tumor size A population-based study in Japan reported lymph node metastasis of $3.7 \%$ for rectal carcinoids with sizes of $5 \mathrm{~mm}$ or less and $9.7 \%$ lymph node metastasis for tumors with sizes of less than $10 \mathrm{~mm}$ $(6,20,21)$. Analysis of the Connecticut Tumor Registry data found that one out of 6 tumors $<2$ $\mathrm{cm}$ was nonlocalized, compared to metastasis in two thirds of patients with tumors $>2 \mathrm{~cm}$. Al Natour and colleagues utilizing the SEER database analyzed predictors of lymph node metastasis based on tumor size and depth of invasion. They found that tumor size (stratifying tumors $<1, \geq 1$ and $<2$, or $\geq 2 \mathrm{~cm}$ ) and depth of invasion (intramucosal, submucosal, or muscularis propria) highly correlated with regional lymph node metastasis Furthermore, patients with intramucosal tumors $<1 \mathrm{~cm}$ had only a $4.0 \%$ rate of lymph node metastasis $(13,14,18,19)$.

Endoscopic ultrasound is excellent for determining exact tumor size and to exclude infiltration of the NETs into the muscular wall (muscularis propria). Endoscopic ultrasound is not mandatory for NETs measuring less than $1 \mathrm{~cm}$, because those do generally not infiltrate the muscular layer.

\section{THERAPEUTIC APPROACHES}

Endoscopic removal, followed by endoscopic surveillance is the treatment of choice in NETs/ carcinoids of the gastrointestinal tract that are $\leq 10 \mathrm{~mm}$ in size, have a low proliferative activity (G1), do not infiltrate the muscular layer and have no angioinvasion. In all the other intestinal NETs, optimal treatment needs surgery and/ or medical therapy depending on type, biology and stage of the tumor $(20,21)$. 
New developments in the endoscopic technology allow early detection of mucosal abnormalities that are amenable to endoscopic. Resection-based modalities consist of endoscopic mucosal resection (EMR) and endoscopic submucosal dissection (ESD). ESD is a newly developed technique that allows en bloc resection of larger (usually more than $20 \mathrm{~mm}$ ) mucosal as well as subepithelial gastrointestinal lesions above the muscularis propria with the use of cutting devices (22).

For tumors with size between 10 and $20 \mathrm{~mm}$ local excision is usually recommended, but radical surgery should be considered if there is evidence of lymph node metastasis or lymphovascular invasion on biopsy. Controversy resides since no established treatment guideline exists. In contrast, radical surgery is recommended for tumors larger than $20 \mathrm{~mm}$, where the risk of metastasis is known to be 60 to $80 \%$. Shields et al. (23) reported that a tumor size of more than $10 \mathrm{~mm}$ and lymphovascular invasion were significantly associated with the presence of nodal disease, rendering mesorectal excision advisable. Cytoreductive surgery is recommended for palliation and to increase survival for patients with advanced disease. To rule out distant metastasis, imaging studies, such as CT or magnetic resonance imaging, are recommended for patients with rectal carcinoids larger than $20 \mathrm{~mm}$ (24).

Surgical treatment, dependent on neoplasm size, site and depth of invasion, is required to resect the primary site of the neoplasm, any sites of metastasis and lymph nodes in order to improve survival rates. Carcinoids of the appendix are often found incidentally during appendectomy. The majority of appendiceal carcinoids are small $(<1 \mathrm{~cm})$, located at the tip of the appendix and often cured with appendectomy. A radical right hemicolectomy is recommended when the primary tumor is $\geq 2 \mathrm{~cm}$, incompletely resected, invades the base of the appendix or meso-appendix, displays lymphovascular invasion, lymph node metastases and unfavorable histology or grade. The need for right hemicolectomy in appendiceal carcinoid of $1-2 \mathrm{~cm}$ in size remains controversial, and decided on a case by case basis. In cecal carcinoids, usually presenting as a bulky mass causing intestinal obstruction or hemorrhage, an oncologic resection is also recommended (25). Cytoreductive surgery should be considered when metastatic disease is localized or if $>70 \%$ of tumor load is thought resectable, which may decrease endocrine and local symptoms and help improving systemic treatment. There are no randomized clinical tri- als comparing the efficacy of locoregional therapies and palliative liver surgery. The choice of the ablative or locoregional procedure such as radiofrequency ablation (RFA), laser-induced thermotherapy or selective hepatic transcatheter arterial embolization (TAE), chemoembolization (TACE) and selective internal radiotherapy (SIRT) depends on the local expertise, number and size of lesions and location of liver involvement. These types of locoregional therapies are usually used in combination with systemic medical treatment $(26,27)$.

An RO situation should be the aim of hepatic surgery, but also patients with R1 or R2 resection show a good survival benefit.

In GEP-NET there is a wide array of other therapeutic options, such as interventional radiology, somatostatin analogues, interferon, peptide-receptor radionuclide therapy, targeted agents (sunitinib, everolimus, bevacizumab) and chemotherapy to palliate symptoms and increase survival (18). Biotherapy and molecular targeted therapy have good prospects in the treatment of patients with progressive NET. Chemotherapy is mainly used to treat patients with NEC or MANEC, while NETs have low sensitivity to chemotherapy

The use of somatostatin analogs is standard therapy in functional NETs of any size. Interferon alpha may also be used for symptoms control in some patients, but is usually used as second line therapy due to its toxic profile. The antitumor efficacy of somatostatin analogs appears weak with respect to objective tumor response (5-10\%). However, disease stabilization of up to $50-60 \%$ has been reported. In a prospective randomized placebo-controlled trial of octreotide long-acting release (LAR), an antiproliferative efficacy has been confirmed. The median time to tumor progression was 14.3 months with octreotide LAR versus 6 months with placebo.

Somatostatin analogs are the recommended first line therapy in functional as well as nonfunctional progressive G1/G2 NETs. In contrast, in metastatic NEC G3 somatostatin analog treatment is not recommended (III, B). Other specific therapies in GEP-NETs are the mTOR-inhibitor everolimus, alone or in combination with a somatostatin analog. In the RADIANT-2 trial which was a randomized phase III trial in patients with NETs (carcinoids), everolimus demonstrated a significant antitumor effect compared with placebo.

Tyrosine kinase inhibitors, sunitinib and pazopanib, have demonstrated significant antitumor efficacy in pancreatic NETs. 
Chemotherapy is recommended in metastatic NET G2 and in NEC G3 of any site. A combination of streptozotocin and 5-fluorouracil (5-FU)/ doxorubicin is indicated in patients with inoperable progressive hepatic metastases from G1/ G2 NETs leading to objective response rates of $35-40 \%$. Chemotherapy using cisplatinum/ etoposide is recommended in cases of highgrade NEC G3 with liver metastases regardless of the site of the primary tumor There is no established second-line therapy for poorly differentiated endocrine carcinoma, but recent retrospective studies have demonstrated the efficacy of temozolomide alone or in combination with capecitabine \pm bevacizumab. Encouraging results have been obtained with 5-FU i.v. or capecitabine orally combined with oxaliplatin or irinotecan $(12,28-30)$.

Promising data have evolved with regard to peptide receptor targeted radiotherapy (PRRT) using 90Yttrium and 177 Lutetium (31) in the treatment of NETs with liver metastases. PRRT may be considered in both functional and nonfunctional NETs with positive somatostatin receptor scintigraphy irrespective of the primary tumor site. Based on phase II trials, the objective response rates range between $20 \%$ and $40 \%$. The highest objective response rate has so far been obtained in metastatic rectal NETs. Prospective randomized trials are still lacking but in progress.

Follow-up investigations should include biochemical parameters and conventional imaging. In cases with R0/R1 resected NET G1/G2 a CT or $M R I$ is recommended every 3-6 months and in NEC G3, every 2-3 months. Somatostatin receptor imaging, either Octreoscan or PET/CT might be included in the follow-up and is recommended after 18-24 months if expression of somatostatin receptor $2 \mathrm{a}$ has been proven in the tumor cells. If chromogranin A is not elevated NSE represents an alternative biomarker (32).

In localized tumors, the 5-year survival is $94 \%$, decreasing to $64 \%$ with regional lymph node involvement and $18 \%$ with distant metastases. An analysis of overall survival stratified by TNM staging (33) revealed that 5 -year survival rates were $100 \%$ for stage I and II tumors vs. $91 \%$ for stage III (locoregionally advanced) and $72 \%$ for stage IV tumors. The median overall survival for stage IV tumors was 103 months. Nodal-negative rectal NETs that are $\leq 1 \mathrm{~cm}$ in size without angioinvasion or infiltration of the muscular layer have an excellent 5-year-survival rate of $98.9-100 \%$. For nodal-positive rectal carcinoid disease (without distant metastases de- tected at the time of diagnosis) the 5-year-survival rate is $54-73 \%$. In rectal NETs with distant metastases, the 5-year-survival rate ranges between $15-30 \%$.

The risk of lymph node metastases of rectal NETs/carcinoids is not lower than the metastatic risk of rectal adenocarcinoma of the same size. Neither is the prognosis of patients with metastatic rectal NET disease better than that of patients suffering from metastastic rectal adenocarcinoma of the same size $(33,34)$. Recent Korean studies suggest that the endoscopic treatment of small $(<10 \mathrm{~mm})$ rectal NETs without evidence of regional or distant metastasis can achieve highly favorable long-term outcomes. In contrast, the risk of recurrence is markedly increased in rectal NET patients with metastatic lymph nodes, even after radical surgery (35).

A particulary group of poor survival neuroendocrine tumors is represented by the highgrade neuroendocrine carcinomas, defined as those having a high mitotic rate $>10$ mitotic figures by 10 high-powered fields, or a Ki-67 proliferative index $>20 \%$. They are managed primarily with platinum-based chemotherapy and have a 5 -year overall survival of $15 \%$ and median survival of 10 months (36)

An important aspect in patients with carcinoid tumors is the increased risk $(13 \%$ to $41 \%)$ of developing a secondary noncarcinoid malignancy compared to the general population. The secreted bioactive agents produce proliferative peptides that may enhance the development of other neoplasia. Most synchronous tumors were observed in the gastrointestinal tract, whereas metachronous tumors were more often observed outside the gastrointestinal tract $(13,37)$.

Colorectal neuroendocrine tumors are reported to have a rate of second primary malignancy in of $5 \%$ to $32 \%$. The most common site of associated non-carcinoid malignancies is the gastrointestinal tract, which involves between 32 and $62 \%$ of all tumors, followed by the genitourinary tract (9 to 22\%) and the lung and/or bronchial system (9 to $13 \%$ ) $(38,39)$.

Data from many studies strongly suggest that when a carcinoid tumor is identified in a patient, there is a need for close surveillance of the gastrointestinal tract, respiratory system and genitourinary tract. Long-term follow-up is recommended for patients in order to identify delayed metastasis and secondary malignancies. The European Neuroendocrine Tumor Society recommends CT and colonoscopy follow-up 
through 10 years, with the frequency of surveillance based on the characteristics of the initial carcinoid tumor $(18,40)$.

\section{CONCLUSIONS}

The gastrointestinal tract constitutes the most common site of extra-pulmonary neuroendocrine tumors. Small colorectal neuroendocrine tumors are effectively treated by local resection, which provides good oncological and surgical outcomes. For larger tumors, rad- ical resection provides acceptable oncological outcomes. Regular surveillance is highly recommended for high-risk patients for long-term management.

The treatment of colon and rectal neuroendocrine tumors is complex one and depends of the individuality of each patient. With adequate management, the prognosis can be favorable with long survival, although related to the tumor differentiation degree, efficacy of the chosen treatment and to the patient adhesion to the follow-up after treatment.

\section{REFERENCES}

1. Hauso O., Gustafsson B.I., Kidd M., Waldum H.L., Drozdov I., et al. Neuroendocrine tumor epidemiology: contrasting Norway and North America. Cancer. 2008; 113:2655-2664

2. Yao J.C., Hassan M., Phan A. et al. One hundred years after "carcinoid": epidemiology of and prognostic factors for neuroendocrine tumors in 35,825 cases in the United States. J Clin Oncol 2008; 26:3063-3072

3. Ballantyne G.H., Savoca P.E., Flannery J.T., et al. Incidence and mortality of carcinoids of the colon. Data from the Connecticut Tumor Registry. Cancer. 1992; 69:2400-2405

4. Jain A., Singla S., Jagdeesh K.S., Vishnumurthy H.Y. Mixed adenoneuroendocrine carcinoma of cecum: a rare entity. J Clin Imaging Sci. 2013; 3:10

5. Pape U.F., Jann H., Muller-Nordhorn J. et al. Prognostic relevance of a novel TNM classification system for upper gastroenteropancreatic neuroendocrine tumors. Cancer 2008; 113:256-265.

6. Edge S.B.B.D., Compton C.C. AJCC Cancer Staging Manual. 7. New York: Springer; 2010

7. Fahy B.N., Tang L.H., Klimstra D., Wong W.D., Guillem J.G., Paty P.B., Temple L.K., Shia J., Weiser M.R. Carcinoid of the rectum risk stratification (CaRRs): a strategy for preoperative outcome assessment. Ann Surg Oncol. 2007; 14:1735-1743

8. Spread C., Berkel H., Jewell L., et al. Colon carcinoid tumors. A population-based study. Dis Colon Rectum. 1994; 37:482-491

9. Waisberg D.R., Fava A.S., Martins L.C., et al. Colonic carcinoid tumors: a clinicopathologic study of 23 patients from a single institution. Arq Gastroenterol. 2009; 46:288-293

10. Weil C. Gastroenteropancreatic endocrine tumors. Klin Wochenschr 1985; 63:433-459
11. Vinik A.I., Woltering E.A., Warner R.R., Caplin M., O'Dorisio T.M., Wiseman G.A., Coppola D., Go V.L. NANETS consensus guidelines for the diagnosis of neuroendocrine tumor. Pancreas 2010; 39:713-734

12. Oberg K., Akerstrom G., Rindi G., Jelic S., Group E.G.W. Neuroendocrine gastroenteropancreatic tumours: ESMO Clinical Practice Guidelines for diagnosis, treatment and follow-up. Ann Oncol. 2010; 21(Suppl 5):v223-227

13. Modlin I.M., Kidd M., Latich I., Zikusoka M.N., Shapiro M.D. Current status of gastrointestinal carcinoids. Gastroenterology. 2005; 128:1717-1751

14. Al Natour R.H., Saund M.S., Sanchez, et al. Tumor size and depth predict rate of lymph node metastasis is colon carcinoids and can be used to select patients for endoscopic resection. J Gastrointest Surg. 2012; 16:595-602

15. Hee Hyun J., Dae Lee S., Youk E.G., Bum Lee J., LeeHee Enu-Jung, Chang J., Dae Kyung Sohn. Clinical impact of atypical endoscopic features in rectal neuroendocrine tumors. World $J$ Gastroenterol. 2015 Dec 21; 21(47): 13302-13308

16. Lloyd R.V. Practical markers used in the diagnosis of neuroendocrine tumors. Endocr Pathol. 2003; 14:293-301

17. Nehar D., Lombard-Bohas C., Olivieri S., Claustrat B., Chayvialle J.A., Penes M.C., Sassolas G., Borson-Chazot F. Interest of Chromogranin A for diagnosis and follow-up of endocrine tumours. Clin Endocrinol (Oxf) 2004; 60:644-652

18. Ramage J.K., Goretzki P.E., Manfredi R., et al. Consensus guidelines for the management of patients with digestive neuroendocrine tumours: well-differentiated colon and rectum tumour/carcinoma. Neuroendocrinology. 2008; 87:31-39

19. NCCN Clinical Practice Guidelines v.1.2012. Neuroendocrine Tumors
20. Akerstrom G., Hellman P. Surgery on neuroendocrine tumours. Best Pract Res Clin Endocrinol Metab 2007; 21:87-109

21. Soga J. Early-stage carcinoids of the gastrointestinal tract: an analysis of 1914 reported cases. Cancer. 2005; 103 : 1587-1595

22. Hulagu S., Senturk O., Aygun C., Kocaman O., Celebi A., Konduk T. et al. Endoscopic submucosal dissection for premalignant lesions and noninvasive early gastrointestinal cancers. World $J$ Gastroenterol. 2011 Apr 7; 17(13): 1701-1709

23. Shields C.J., Tiret E., Winter D.C. Carcinoid tumors of the rectum: a multiinstitutional international collaboration. Ann Surg. 2010; 252:750-755

24. Garcia-Carbonero R., Salazar R., Sevilla I., Isla D. SEOM clinical guidelines for the diagnosis and treatment of gastroenteropancreatic neuroendocrine tumours (GEP NETS) Clin Transl Oncol. 2011; 13(8):545-551

25. Kunz P.L., Reidy-Lagunes D., Anthony L.B., Bertino E.M., Brendtro K., Chan J.A., Chen H., Jensen R.T., Kim M.K., Klimstra D.S., Kulke M.H., Liu E.H., Metz D.C., Phan A.T., Sippel R.S., Strosberg J.R., Yao J.C. Consensus guidelines for the management and treatment of neuroendocrine tumors. Pancreas. 2013; 42:557-577

26. Sarmiento J.M., Que F.G. Hepatic surgery for metastases from neuroendocrine tumors. Surg Oncol Clin N Am 2003; 12:231-242

27. Kennedy A.S., Warner R. Radioembolisation for neuroendocrine hepatic metastases. Cancer Investigation 2007; 25:55-56

28. Turner N.C., Strauss S.J., Sarker D. et al. Chemotherapy with 5-fluorouracil, cisplatin and streptozocin for neuroendocrine tumours. Br J Cancer 2010; 102: 1106-1112. 
29. Sorbye H., Welin S., Langer S. et al. NNTG: Nordic Neuroendocrine Tumor Group. Predictive and prognostic factors for treatment and survival in 305 patients with advanced gastrointestinal poorly differentiated neuroendocrine carcinoma: The NORDIC NEC study. ASCO 2012

30. Rinke A., Muller H.H., Schade-Brittinger C. et al. Placebo-controlled, double-blind, prospective, randomized study on the effect of octreotide LAR in the control of tumor growth in patients with metastatic neuroendocrine midgut tumors: a report from the PROMID Study Group. J Clin Oncol. 2009; 27:4656-4663

31. Kwekkeboom D.J., de Herder W.W., Kam B.L. et al. Treatment with the radiolabeled somatostatin analog [177 LuDOTA 0,Tyr3] octreotate: toxicity, efficacy, and survival. J Clin Oncol 2008; 26: 2124-2130

32. Öberg K., Knigge U., Kwekkeboom D. Neuroendocrine gastro-entero-pancreatic tumors: ESMO Clinical Practice Guidelines for diagnosis, treatment and follow-up. Annals of Oncology 23 (Supplement 7): 2012; vii124 - vii130
33. Jann H., Roll S., Couvelard A., Hentic O., Pavel M., Muller-Nordhorn J., Koch M., Rocken C., Rindi G., Ruszniewski P., Wiedenmann B., Pape U.F. Neuroendocrine tumors of midgut and hindgut origin: tumor-node-metastasis classification determines clinical outcome. Cancer. 2011; 117:3332-3341

34. Konishi T., Watanabe T., Kishimoto J., Kotake K., Muto T., Nagawa H. Prognosis and risk factors of metastasis in colorectal carcinoids: results of a nationwide registry over 15 years. Gut. 2007; 56:863-868

35. Kim D.H., Lee J.H., Cha Y.J, et al. Surveillance strategy for rectal neuroendocrine tumors according to recurrence risk stratification. Dig Dis Sci. 2014; 59:850-856

36. Smith J.D., Reidy D.L., Goodman K.A., Shia J., Nash G.M. A Retrospective Review of 126 High-Grade Neuroendocrine Carcinomas of the Colon and Rectum. Ann Surg Oncol. 2014 Sep; 21(9): 2956-2962.

37. Maroun J., Kocha W., Kvols L., et al. Guidelines for the diagnosis and management of carcinoid tumours. Part 1: The gastrointestinal tract. A statement from a Canadian National Carcinoid Expert Group. Curr Oncol. 2006; 13:67-76

38. Li A.F., Hsu C.Y., Li A., Tai L.C., Liang W.Y., Li W.Y., Tsay S.H., Chen J.Y. A 35-year retrospective study of carcinoid tumors in Taiwan: differences in distribution with a high probability of associated second primary malignancies. Cancer. 2008; 112:274-283

39. Tichansky D.S., Cagir B., Borrazzo E., Topham A., Palazzo J., Weaver E.J., Lange A., Fry R.D. Risk of second cancers in patients with colorectal carcinoids. Dis Colon Rectum. 2002; 45:91-97

40. Anthony L.B., Strosberg J.R., Klimstra D.S., Maples W.J., O'Dorisio T.M., Warner R.R., et al. The NANETS consensus guidelines for the diagnosis and management of gastrointestinal neuroendocrine tumors (nets): well-differentiated nets of the distal colon and rectum. Pancreas. 2010; 39:767-74 